\title{
Criminologie
}

\section{Le taxage : une forme inédite de vol ?}

\section{Marc Le Blanc et Anne-Élyse Deguire}

Volume 35, numéro 2, automne 2002

Femmes et enfermement au Canada : une décennie de réformes

URI : https://id.erudit.org/iderudit/008296ar

DOI : https://doi.org/10.7202/008296ar

Aller au sommaire du numéro

Éditeur(s)

Les Presses de l'Université de Montréal

ISSN

0316-0041 (imprimé)

1492-1367 (numérique)

Découvrir la revue

Citer cet article

Le Blanc, M. \& Deguire, A.-É. (2002). Le taxage : une forme inédite de vol ? Criminologie, 35(2), 159-178. https://doi.org/10.7202/008296ar

\section{Résumé de l'article}

Il existe beaucoup de confusion concernant la définition du taxage et son ampleur chez les adolescents, ceci à la fois dans la communauté scientifique et dans la société en général. Certains font du taxage un geste qui découle directement de la participation à un sous-groupe déviant de la culture adolescente, d'autres considèrent cette activité comme un geste délinquant parmi d'autres. Pour tenter de clarifier ces interprétations du taxage, cet article rapporte une enquête auprès d'adolescents qui représentent la population. Ils ont été interrogés sur leur participation à six formes de taxage et, tout particulièrement, sur les caractéristiques de ces actes. Il ressort que la participation au taxage, comme auteur ou victime, concerne un très petit nombre d'adolescents, des garçons surtout. C'est un acte délinquant qui n'est pas particulièrement associé à la fréquentation scolaire, qui est commis en groupe et dont la gravité est habituellement légère. Le taxage devient rarement un acte complexe par les méthodes utilisées (menace, violence physique et utilisation d'une arme) et grave par la valeur des biens extorqués ou des droits de passage exigés. En somme, le taxage se manifeste davantage comme un vol que comme un geste qui émane de la différenciation de la sous-culture adolescente en sous-groupes ou du caïdage.
Ce document est protégé par la loi sur le droit d'auteur. L’utilisation des services d’Érudit (y compris la reproduction) est assujettie à sa politique d'utilisation que vous pouvez consulter en ligne.

https://apropos.erudit.org/fr/usagers/politique-dutilisation/ 


\title{
Le taxage : une forme inédite de vol?
}

\author{
Marc Le Blanc \\ Professeur \\ École de psychoéducation \\ École de criminologie \\ Université de Montréal \\ marc.leblanc@umontreal.ca \\ Anne-Élyse Deguire \\ Spécialiste en prévention \\ Centre international d'étude sur le jeu \\ et les comportements à risque chez les jeunes \\ Université McGill
}

RÉSUMÉ - Il existe beaucoup de confusion concernant la définition du taxage et son ampleur chez les adolescents, ceci à la fois dans la communauté scientifique et dans la société en général. Certains font du taxage un geste qui découle directement de la participation à un sous-groupe déviant de la culture adolescente, d'autres considèrent cette activité comme un geste délinquant parmi d'autres. Pour tenter de clarifier ces interprétations du taxage, cet article rapporte une enquête auprès d'adolescents qui représentent la population. Ils ont été interrogés sur leur participation à six formes de taxage et, tout particulièrement, sur les caractéristiques de ces actes. Il ressort que la participation au taxage, comme auteur ou victime, concerne un très petit nombre d'adolescents, des garçons surtout. C'est un acte délinquant qui n'est pas particulièrement associé à la fréquentation scolaire, qui est commis en groupe et dont la gravité est habituellement légère. Le taxage devient rarement un acte complexe par les méthodes utilisées (menace, violence physique et utilisation d'une arme) et grave par la valeur des biens extorqués ou des droits de passage exigés. En somme, le taxage se manifeste davantage comme un vol que comme un geste qui émane de la différenciation de la sous-culture adolescente en sous-groupes ou du caïdage.

ABSTRACT - There is much confusion, in the scientific community and in society in general, about the definition of taxing and its prevalence among adolescents. Taxing is either associated with the participation in a particular deviant subculture or as part of the general deviant syndrome. In this paper, a survey is used to clarify the nature of taxing during adolescence. Six forms of taxing are described, particularily the characteristics of the perpetration of this behavior. It was observed that a very small proportion of adolescents are part, as victims or authors, of acts of taxing, more so for boys. This 
behavior is not particularily associated with schooling; it is perpetrated in groups and it is not a sophisticated or serious crime. Very rarely, there is use of violence and weapons and the value of the tax is usually low. In sum, taxing appears more as a form of delinquency then a behavior that characterize a deviant adolescent subculture.

\section{Introduction}

1953 : Marc, 10 ans, circule sur le trottoir dans une rue adjacente de chez lui. Il vient d'acquérir son premier gant et une balle de baseball neuve. Deux adolescents l'accostent et les lui demandent. Il résiste timidement. Ils s'en emparent et se sauvent.

Il y a un demi-siècle, un événement semblable aurait été qualifié de vol. Aujourd'hui, le terme de «taxage» est utilisé pour décrire cet événement. Les médias traitent régulièrement du phénomène du taxage qu'ils associent à la fréquentation de l'école. Ce terme est employé autant par les adolescents, les parents, les autorités scolaires, les enseignants et les intervenants sociaux. Tous définissent le taxage comme un comportement violent pratiqué dans le dessein d'extorquer de l'argent ou des biens. En Europe, c'est le terme de racket qui est employé pour décrire le même genre d'événement (Buidin et al., 2000; Debarbieux, 1996; 2001).

Il est bien connu que la criminalité de violence des adolescents a augmenté considérablement entre la fin des années 1980 et le milieu des années 1990, spécialement sous la forme de l'accroissement du nombre d'actes violents commis par un groupe très restreint d'adolescents (Le Blanc, 1999). Ainsi, les actes décrits ci-dessus seraient commis le plus souvent par des adolescents, le taxage, une forme de criminalité avec violence, étant devenu, avec raison, une préoccupation dans plusieurs sociétés occidentales. Pour la France, Debarbieux (1996) rapporte que l'évolution des rackets scolaires a suivi celle de la délinquance juvénile en général : diminution de 1982 à 1986, augmentation légèrement plus forte que celle de l'ensemble de la délinquance de 1988 à 1992, et diminution en 1993.

Au Québec, le journal La Presse (13-14-15 décembre 1998) affirmait que $80 \%$ des adolescents étaient impliqués d'une manière ou d'une autre dans le taxage. Cette forme de conduite délinquante serait le reflet d'une société de consommation de plus en plus violente. Néanmoins, quiconque s'intéresse à ce phénomène rencontre un obstacle de taille. Le taxage n'a pas fait l'objet d'une analyse conceptuelle conduisant à une définition opérationnelle et il n'a pas fait non plus l'objet d'une étude empirique d'envergure caractérisée par l'utilisation d'un échantillon représentatif d'adolescents ni non plus d'une analyse en profondeur du pas- 
sage à l'acte du point de vue de l'auteur et de la victime. Cet article veut combler ces lacunes.

\section{Le taxage, une forme de vol?}

Le terme de «taxage» est absent des dictionnaires de langue française, tels le Robert et le Larousse. Il provient toutefois du nom «taxe» et du verbe «taxer». Le premier est un impôt sur quelque chose et le second est l'action de l'imposer d'autorité; c'est habituellement le rôle de l'État ou d'un organisme institutionnel. Le fait de devoir donner de l'argent ou un bien sous la menace, est-ce du taxage ou du racket? À première vue, ce dernier terme est plus approprié parce que les dictionnaires le définissent comme de l'extorsion d'argent par le chantage, l'intimidation ou la terreur. Toutefois, le racket est décrit comme le fait de malfaiteurs ou de gangs de délinquants, ce qui s'applique peut-être moins aux adolescents. Le taxage est vraisemblablement une forme juvénile des rackets qui sont mis en place par des adultes criminels.

Au Québec, selon une revue des médias qui traitent de la question (Actualités Québec, 1999), le taxage fait référence, dans le vocabulaire populaire, à une forme de vol où des adolescents soutirent à d'autres adolescents des blousons, des souliers de course ou de l'argent par la menace et l'intimidation; parfois, cette action est accompagnée de violence physique. Cette définition est reprise et élargie par Bianki et Lampron (1998). Ils caractérisent le taxage comme un rapport de force ou un abus qui est pratiqué principalement par des adolescents; ceux-ci convoitent des objets, symboliques ou non, dans des endroits publics fréquentés par leurs pairs, principalement à l'école, en utilisant des degrés de force variés, allant de la menace à l'utilisation d'une arme. Ces auteurs considèrent donc le taxage comme une manifestation de la culture adolescente. Le taxage devient ainsi une forme nouvelle de vol et d'extorsion.

La culture adolescente est constituée d'agglomérations d'individus qui sont stéréotypés par leurs comportements, leurs attitudes, leur tenue vestimentaire ou d'autres caractéristiques. À Montréal, par exemple, ces sous-cultures seraient les «preppies», les «yos», les «sportifs», les «alternatifs», les «grunges», les «raveux», les «gothiques», les «skinheads», les «punks», les «ginos», les «nerds», les «poils» ou «heavy metal», sans oublier les «conventionnels», qui n'appartiennent pas à une sousculture dans le sens propre du terme et qui sont identifiés comme tels par leurs pairs. Par ailleurs, il importe de distinguer les sous-cultures qui 
émergent en réponse aux exigences sociales et culturelles dominantes, de celles qui apparaissent en réaction à celles-ci. C'est ainsi qu'il est possible de distinguer, parmi les premières, les adolescents conventionnels et les adolescents sur-conventionnels (par exemple, les «nerds») et, parmi les secondes, les adolescents asociaux (par exemple, les «gothiques», les «skinheads», les «punks», etc.).

Un adolescent peut vivre cette période développementale de différentes façons, selon le groupe auquel il appartient. Il peut être satisfait de son destin ou aspirer à quelque chose de prestigieux, à ses yeux et aux yeux de ses pairs. Certaines des modalités liées à la façon de vivre l'adolescence peuvent avoir un impact sur le fait de s'adonner au taxage. Ainsi, en quête d'un statut, d'une identité ou pour faire partie d'une sousculture, un adolescent pourrait en venir à «taxer» des biens qui sont identifiés à cette sous-culture, puisqu'il ne dispose pas des moyens légitimes pour les obtenir. Le taxage devient alors un moyen pour s'identifier à une sous-culture et pour faire partie intégrante d'une clique qui en relève. Par ailleurs, si cet adolescent vit des sentiments de rejet et de dépréciation en regard de cette sous-culture, il pourrait se regrouper avec d'autres qui partagent son expérience. Ceux-ci s'adonneront alors à des activités de taxage sur les membres d'une autre sous-culture, soit pour d'obtenir les biens désirés, soit pour remédier à leur sentiment d'infériorité ou s'affirmer dans leur rôle. C'est également dans ce cas que le taxage peut se manifester sous forme d'extorsion d'argent.

En criminologie, le taxage peut se rapprocher $\mathrm{du}$ «mugging», mais il doit être différencié du caïdage. Lejeune (1977) définit le premier comme l'action de voler ou de prendre quelque chose en la présence de son propriétaire en utilisant la force ou la menace; il est commis dans des endroits publics et semi-publics par des agresseurs plus jeunes et davantage inexpérimentés. Le second consiste plutôt, selon Olweus (1993), à tyranniser quelqu'un, à provoquer de la douleur et une certaine détresse chez sa victime, habituellement sans appropriation de ses biens; ce comportement a un caractère répétitif et il est habituellement le fait d'enfants plutôt que d'adolescents.

Pour sa part, le droit pourrait associer le taxage à une forme de vol qualifié, puisque le Code criminel canadien en propose la définition suivante :

Commet un vol qualifié quiconque, selon le cas : a) vole et, pour extorquer la chose volée ou empêcher ou maîtriser toute résistance au vol, emploie la violence ou des menaces de violence contre une personne ou des biens; 
b) vole quelqu'un et, au moment où il vole, ou immédiatement avant ou après, blesse, bat ou frappe cette personne ou se porte à des actes de violence contre elle; c) se livre à des voies de fait sur une personne avec l'intention de la voler; d) vole une personne alors qu'il est muni d'une arme offensive ou d'une imitation d'une telle arme (art. 343, Code Criminel annoté et lois connexes, 1991).

Dans le cadre de cette définition, Fréchette et Le Blanc (1987), en demandant aux adolescents de raconter leurs délits, décrivent deux formes de vol qui s'apparentent au taxage, car leur but est de s'approprier des biens à l'aide de moyens coercitifs exercés directement sur la victime. Il s'agit du vol grave et du vol d'une personne. Le premier se caractérise par l'utilisation de moyens physiques par le délinquant pour atteindre son but, alors que le second se démarque par une pression psychologique, le délinquant préférant alors l'utilisation des menaces et de l'intimidation. Poupart (1996) reconnaît que le taxage est une forme de vol qualifié commis avec une intention d'extorsion, d'intimidation et de harcèlement.

Deux perspectives s'imposent donc pour définir le taxage chez les adolescents. D'une part, le taxage serait une forme nouvelle de délinquance avec violence parce qu'il émane de la compétition entre plusieurs sous-cultures adolescentes; il s'agit d'un vol de biens valorisés par l'un ou l'autre sous-groupe d'adolescents. D'autre part, le taxage ne serait qu'une des formes que prend le vol qualifié. Ce qui distingue ces deux perspectives, c'est la motivation. Dans le premier cas, elle prend sa source dans les mécanismes de socialisation qui sont propres à l'adolescence. Dans le second cas, les motivations varient selon les individus. Il est difficile de donner raison à l'une ou l'autre perspective sans une analyse exhaustive du passage à l'acte. Cette analyse permettra de vérifier si le taxage est avant tout un geste qui permet de s'approprier des biens symboliques associés à une sous-culture adolescente ou si le taxage est tout simplement une forme de vol qualifié qui est comparable aux autres formes de vols d'une personne.

Puisque le taxage est un geste délinquant, voyons comment il se manifeste. Compte tenu de l'absence d'études empiriques adéquates sur le taxage, le présent article se penche sur trois questions principales : Quelle est la prévalence du taxage selon les auteurs et les victimes? Est-ce que le phénomène du taxage varie selon le sexe, l'âge, l'origine ethnique, le désavantage socioéconomique et le cheminement scolaire? Comment se manifestent les modalités du passage à l'acte de taxage? 


\section{Esquisse de recherche}

Cette étude porte sur un échantillon de 402 adolescents, 198 filles et 204 garçons, parmi les 1034 élèves d'un établissement d'études secondaires de la région métropolitaine. Il sont âgés de 12 à 19 ans et inscrits à temps plein dans cette école polyvalente. Cet établissement occupe une position médiane parmi l'ensemble des établissements selon un indice de désavantage socioéconomique (Conseil scolaire de Montréal, 1999). Parmi ces 402 élèves, 349 élèves font partie de classes régulières, dont 79 en première année du secondaire, 82 en deuxième année, 71 en troisième année, 64 en quatrième année et 53 en cinquième année du secondaire. De plus, deux classes «d'accélération» ont été sélectionnées, soit 53 élèves; elles offrent un service particulier aux étudiants qui ont accumulé un certain retard académique, plus particulièrement des adolescents pris en charge par les Centres jeunesse. Les 402 adolescents forment $59 \%$ de la clientèle de cette école régulière. Une école spéciale a ensuite été sélectionnée; elle dessert une clientèle ayant été référée par le système scolaire et les Centres jeunesse de Montréal. Il s'agit de 38 garçons et de 40 filles.

Tous ces adolescents ont répondu à la forme auto-administrée du MASPAQ (Mesures de l'Adaptation Sociale et Personnelle pour les Adolescents Québécois) (Le Blanc, 1996). Cet instrument inventorie la conduite délinquante et les troubles de comportement, le fonctionnement de la famille, l'expérience scolaire, les activités routinières, les relations avec les pairs, les attitudes relatives aux normes de conduite et la personnalité. Les propriétés métriques des échelles qui composent le MASPAQ sont documentées (Le Blanc, 1996). Pour les fins de cette étude, le MASPAQ a été enrichi de questions relatives au taxage (voir en annexe).

Les questions sur le taxage ont pris la même forme que les questions sur les conduites déviantes du MASPAQ: avoir déjà eu un comportement donné, l'âge auquel le comportement a été adopté pour la première fois et la fréquence du comportement au cours de la dernière année. Les questions sur le taxage étaient présentées sous la forme révélée (l'avoir fait) et la forme subie (avoir été victime). Dans chaque cas, il s'agit de six questions : trois questions sur les objets extorqués (par la menace, par des coups, par l'utilisation d'une arme) et trois questions sur l'argent soutiré en échange d'un droit de passage (par la menace, par des coups, par l'utilisation d'une arme). Ensuite, les adolescents qui ont rapporté avoir commis ou subi un acte de taxage au cours des douze derniers mois devaient répondre à des questions sur les circonstances entourant ce geste, par 
exemple le lieu où l'acte avait été commis, la présence de complices, etc. Finalement, pour déterminer le lien entre l'acte de taxage et la sous-culture adolescente, des questions concernaient l'identification de l'adolescent à une composante de la sous-culture adolescente, ainsi que le groupe d'appartenance du taxeur et du taxé. Toutes ces questions sont en annexe.

Deguire (2000) décrit en détail toutes ces questions et les échelles qui en découlent. Elle rapporte les propriétés métriques de ces mesures, plus spécifiquement des coefficients de fidélité (Alpha de Cronbach) qui varient de 0,83 à 0,94 . De plus, la validité est intéressante, puisqu'il y a une corrélation statistiquement significative $(p=0,0001)$ de 0,38 entre l'échelle sur le taxage rapporté et l'échelle sur la délinquance criminelle du MASPAQ, celle-ci ne comprenant pas de comportements pouvant être associés au taxage.

\section{Résultats}

La participation au taxage

Parmi les adolescents interrogés, 13,6\% révèlent s'être adonnés à une activité de taxage au cours de leur vie, mais seulement 9,2\% avouent s'y être adonnés au cours des douze derniers mois. Ces derniers ont pratiqué deux des six formes de taxage pendant cette période (moyenne de 2,6 et écart type de 1,9), mais ces comportements étaient peu fréquents (moyenne de 9,8 et écart type de 3,9 pour une échelle dont le minimum est de 6 et le maximum de 24). Si les taxeurs constituent une faible minorité des adolescents, ils paraissent légèrement plus nombreux selon les réponses des victimes. En effet, 21,9\% des adolescents révèlent avoir été taxés au moins une fois au cours de leur vie, alors que $11,1 \%$ rapportent avoir été taxés au cours des douze derniers mois. Parmi ces adolescents taxés, très peu sont victimes à répétition, puisqu'ils font généralement l'objet d'une seule forme de taxage sur les six formes investiguées (moyenne de 1,9 et écart type de 1,3) et cela rarement (fréquence moyenne de 8 et écart type de 1,65 pour un minimum de 6 et un maximum de 24). Cette légère différence entre le niveau de prévalence selon les taxeurs et les taxés s'explique par le fait que les taxeurs sont moins nombreux, mais que plusieurs d'entre eux le font à répétition. Par contre, les taxés ne sont habituellement victimes qu'une seule fois.

Le taux de victimisation cumulative de $21 \%$ se rapproche du pourcentage d'adolescents victimes de vol sur la personne à l'école de Kuther et 
Fisher (1998), qui était de $18 \%$. Par ailleurs, le taux de victimisation récente, de $11 \%$, est comparable à celui du vol sur la personne qui ne dépasse pas $12 \%$, selon Garofalo, Siegel et Laub (1987) et Kingery, Coggeshall et Alford (1998). Par contre, ce pourcentage de victimisation récente est supérieur à ceux rapportés dans des enquêtes européennes. Debarbieux, Dupuch et Montaya (1997) indiquent que 9\% des lycéens disent avoir été victimes de racket scolaire en France; $8 \%$ selon les adultes œuvrant en milieu scolaire (Debarbieux, 1996); Buidin et al. (2000) obtiennent, pour leur part, $5 \%$ de victimes de taxage en Belgique. Il convient toutefois de souligner que cette différence peut, en partie, résulter de la façon de mesurer la victimisation, le nombre et la nature des comportements variant d'une enquête à l'autre. Par exemple, l'enquête belge n'utilise qu'une question sur le taxage, alors que notre échelle en comporte six, ce qui augmente la possibilité de se rappeler au moins un acte de taxage.

Si le niveau de victimisation doit être comparé avec circonspection, il est encore plus difficile de le faire pour la proportion des adolescents qui taxent, parce que les études sont moins nombreuses. Ainsi, Debarbieux et al. (1997) rapportent que $9 \%$ des lycéens se déclarent auteurs d'actes de racket scolaire, ce qui correspond à notre chiffre de $9 \%$. Cette proportion est comparable à ce que nous disent les statistiques officielles. Selon Statistique Canada, entre $7 \%$ et $11 \%$ des accusations portées contre des mineurs en 1995 concernaient des vols qualifiés. Par contre, ce pourcentage est supérieur à celui qui est rapporté par Fréchette et Le Blanc (1987) pour les vols graves, dans un échantillon d'adolescents conventionnels, au cours des années 1970 (4,4\%). Toutefois, il est très inférieur à celui qui est rapporté pour leur échantillon d'adolescents judiciarisés; en effet, $25 \%$ avouaient avoir commis un vol sur une personne.

En somme, le taxage, selon les auteurs et les victimes, est un comportement délinquant qui n'est pas généralisé, contrairement à ce que laissaient entendre les médias québécois. Une très faible proportion des adolescents ont participé à cette activité au cours de la dernière année ou au cours de leur vie. Est-ce que ces proportions varient selon certaines caractéristiques sociodémographiques des adolescents? Le tableau 1 rapporte la prévalence du taxage selon le sexe, l'âge, l'origine géographique, le désavantage socioéconomique, ainsi que le type de classe fréquenté pour les taxeurs.

Il se dégage du tableau 1 que la participation cumulative des taxeurs varie de façon statistiquement significative selon le sexe $\left(X^{2}=14,29\right.$; $p=0,000)$, l'âge $\left(X^{2}=8,50 ; p=0,014\right)$, l'origine géographique $\left(X^{2}=\right.$ $5,48 ; p=0,019)$ et le type de classe $\left(X^{2}=3,91 ; p=0,048\right)$. Par ailleurs, 
Deguire (2000) rapporte que la participation actuelle varie seulement en fonction du sexe $\left(X^{2}=18,84 ; p=0,000\right)$, alors que la variété et la fréquence du taxage ne varient pas de façon statistiquement significative selon les caractéristiques des taxeurs. Ces résultats indiquent que les taxeurs se recrutent le plus souvent parmi les garçons $(19,5 \%$ chez les garçons comparativement à 7,7\% chez les filles) et chez les 15 et 16 ans $(18,6 \%$ contre $9 \%$ respectivement, chez les plus jeunes et les plus âgés). De plus, les taxeurs sont plus souvent des personnes dont l'origine géographique est à l'extérieur de l'Amérique du Nord (16,7\% compa-

TABLEAU 1

Participation au taxage selon les taxeurs

\begin{tabular}{|l|c|c|c|}
\hline \multirow{2}{*}{} & \multicolumn{2}{|c|}{ Taxage } & \multirow{2}{*}{ Total } \\
\cline { 2 - 3 } Sexe $\left(X^{2}: 14,29 p 0,00\right)$ & & Non & \\
Garçons & $47(19,5 \%)$ & $194(80,5 \%)$ & $241(50,5 \%)$ \\
Filles & $18(7,6 \%)$ & $218(92,4 \%)$ & $236(49,5 \%)$ \\
Total & $65(13,6 \%)$ & $412(86,4 \%)$ & $477(100 \%)$ \\
\hline Âge $\left(X^{2}: 8,5 p 0,01\right)$ & & & \\
Début adolescence & $16(9,5 \%)$ & $152(90,5 \%)$ & $168(35,2 \%)$ \\
Milieu adolescence & $41(18,6 \%)$ & $180(81,4 \%)$ & $221(46,3 \%)$ \\
Fin adolescence & $8(9,1 \%)$ & $80(90,9 \%)$ & $88(18,4 \%)$ \\
Total & $65(13,6 \%)$ & $412(86,4 \%)$ & $477(100 \%)$ \\
\hline Origine géographique & & & \\
$\left(X^{2}: 1,5 p 0,51\right)$ & $18(10,2 \%)$ & $177(90,8 \%)$ & $195(41 \%)$ \\
Amérique du Nord & $47(16,7 \%)$ & $234(83,3 \%)$ & $281(59 \%)$ \\
Ailleurs & $65(13,7 \%)$ & $411(86,3 \%)$ & $476(100 \%)$ \\
Total & & & \\
\hline Désavantage socioéconomique & & & \\
$\left(X^{2}: 1,68 p 0,45\right)$ & $11(8,5 \%)$ & $118(91,5 \%)$ & $129(27 \%)$ \\
Aucun & $65(13,6 \%)$ & $412(86,4 \%)$ & $477(100 \%)$ \\
Peu & $18(13,1 \%)$ & $119(86,9 \%)$ & $137(30,4 \%)$ \\
Désavantagé & $31(12,5 \%)$ & $217(87,5 \%)$ & $248(55,1 \%)$ \\
Total & $12(18,5 \%)$ & $53(81,5 \%)$ & $65(14,4 \%)$ \\
\hline Type de classes & $61(13,6 \%)$ & $389(86,4 \%)$ & $450(100 \%)$ \\
Régulières & & & \\
Spéciales & & & \\
Total & & & \\
\hline
\end{tabular}


rativement à $10,2 \%$ ) et ils fréquentent davantage les classes régulières $(15,5 \%)$ que les classes spéciales $(8,5 \%)$. Par contre, les taxeurs ne proviennent pas plus que les autres de familles désavantagées socioéconomiquement.

En somme, on peut décrire les taxeurs comme étant plus souvent de genre masculin, âgés de 15 et 16 ans, fréquentant une classe régulière et provenant de l'extérieur de l'Amérique du Nord. Par contre, les taxés se décrivent par une seule caractéristique : ce sont le plus souvent des garçons. Cette concentration du taxage par des garçons et sur des garçons est tout à fait conforme aux connaissances criminologiques sur la répartition de la délinquance selon le genre (Lanctôt et Le Blanc, 2002). Par exemple, Fréchette et Le Blanc (1987) observent que les garçons commettent cinq fois plus d'actes délinquants graves que les filles. Une autre caractéristique intéressante du phénomène du taxage est le fait que ce sont le plus souvent des adolescents de 15 et 16 ans qui victimisent d'autres adolescents, quel que soit leur âge. Ainsi, le taxage est surtout une activité délinquante du milieu de l'adolescence et qui apparaît rarement avant ou après cette période. Elle constitue peut-être un tremplin vers des activités délinquantes plus graves contre les personnes. En effet, Fréchette et Le Blanc (1987) montrent que les infractions les plus graves contre la propriété et les personnes apparaissent le plus souvent vers la fin de l'adolescence. L'analyse du passage à l'acte permettra peut-être d'éclairer cette hypothèse.

\section{Les caractéristiques du passage à l'acte}

Il s'agit de décrire les circonstances du geste de taxage selon la méthode proposée par Fréchette et Le Blanc (1987). La nature de l'acte sera d'abord considérée. Les méthodes utilisées seront ensuite décrites, ainsi que la complicité. Dans cette section, nous ne présenterons que les données du passage à l'acte selon le point de vue de l'auteur du taxage. Les données concernant le point de vue de la victime sont analysées par Deguire (2000) et elles concordent tout à fait avec celles de l'auteur. Lorsque des différences entre les points de vue de la victime et de l'auteur apparaitront, nous en ferons mention. De plus, nous nous limiterons aux données sur le taxage récent. En effet, ces données coïncident avec celles qui sont rapportées sur la participation cumulative au taxage (Deguire, 2000). 


\section{La nature du taxage}

Selon certaines définitions du taxage, il s'agit d'un acte qui résulte de la participation à la sous-culture adolescente; plus spécifiquement, il est commandé par une de ses manifestations particulières. En conséquence, le taxage devrait d'abord prendre la forme de l'extorsion d'objets prestigieux. Ensuite, cet acte devrait être commandé par l'appartenance à un sous-groupe qui compose la mosaïque de la sous-culture adolescente. Si ces deux conditions ne sont pas respectées, le taxage devient un vol qualifié comme les autres.

Si 8,6\% des adolescents avouent avoir taxé au cours de la dernière année, la majorité d'entre eux $(5,1 \%)$ se sont limités à un objet; aucun adolescent n'a préféré un droit de passage $(0,5 \%$ pour la participation cumulative) et 3,5\% ont participé à ces deux formes de taxage. Ainsi, $59 \%$ des taxeurs se limitent à des objets, comparativement à $41 \%$ qui taxent un objet ou de l'argent selon les circonstances.

La fréquence des objets taxés ainsi que la valeur des droits de passage, si on s'en tient à ce qu'ont dit les taxeurs, se présentent ainsi (les résultats selon les taxés sont tout à fait comparables selon Deguire, 2000). Parmi les objets taxés, 4,6\% sont prestigieux. Il s'agit d'objets pour lesquels la marque de commerce est précisée par le taxeur, que ce soient des manteaux, des casquettes, des souliers ou tout autre vêtement (par exemple des souliers Nike). Les objets non prestigieux sont trois fois plus nombreux (16,9\%). Il s'agit d'objets pour lesquels la marque de commerce n'était pas connue, donc peu significative pour le taxeur; cette catégorie comprend également des objets dont la valeur monétaire est petite (par exemple des stylos, des confiseries, etc.). Pour leur part, les objets dont la valeur monétaire est élevée sont quatre fois plus nombreux que les objets prestigieux (21,5\%). Il s'agit ici de bijoux, de cigarettes, de drogue, de cartes d'autobus, de baladeurs ou d'autres appareils électroniques (cellulaire, "gameboy», etc.), d'articles de sport et de bicyclettes. L'argent compte pour $24,6 \%$ de ce qui est taxé et le montant est de moins de $20 \$$, dans $35,8 \%$ des cas, et de plus de $20 \$$, dans $35,7 \%$ des cas; dans les autres cas $(14,3 \%)$, le montant n'est pas précisé, ou bien il s'agit de la totalité de l'argent que la personne avait sur elle $(14,3 \%)$. Finalement, $32,1 \%$ des taxeurs sont incapables de préciser ce qu'ils ont obtenu lors de leurs activités de taxage.

En somme, l'argent et les objets d'une valeur monétaire élevée sont le plus souvent ce que les taxeurs convoitent et obtiennent; rarement il s'agit 
d'objets qui caractérisent l'un ou l'autre sous-groupe de la sous-culture adolescente par leur caractère prestigieux ou comme symbole d'appartenance. Par conséquent, l'hypothèse selon laquelle le taxage serait avant tout le résultat de l'identification à un groupe particulier de la sous-culture adolescente ne peut pas expliquer de façon satisfaisante l'ensemble du phénomène : elle ne peut rendre compte que d'une très faible proportion des gestes de taxage. Cette conclusion est confirmée par les données fournies par Deguire (2000) concernant la distribution des objets taxés et la valeur des droits de passage selon les adolescents taxés, puisque cette distribution est semblable pour les différents adolescents taxés.

\section{La présence de sous-cultures chez les taxeurs}

Avant de relier les données sur l'appartenance à une sous-culture, quelques précisions s'imposent en ce qui a trait aux différentes catégories de souscultures utilisées dans cette étude. La première catégorie, les conventionnelles, n'est pas, à proprement parler, une sous-culture. Elle représente surtout les adolescents qui se fondent dans la culture dominante : ils représentent $71,2 \%$ de l'échantillon. Elle regroupe les individus qui s'identifient comme étant soit conventionnels, «preppies», «sportifs» et «nerds». La seconde catégorie, la sous-culture déviante-culturelle, représente les adolescents qui s'identifient comme étant marginaux sans toutefois représenter un danger pour la collectivité. Cette catégorie regroupe les «yos», les «alternatifs», les «grunges», les «raveux», les «gothiques» et les «gino». Ils constituent $9,9 \%$ de l'échantillon. La troisième catégorie, la sous-culture déviante-délinquante regroupe les «skinheads», les «punks», les «poils» ou «heavy metal» et les membres de gangs de rue. Ces adolescents s'identifient comme étant asociaux et potentiellement dangereux pour la collectivité : ils composent $9,1 \%$ de l'échantillon. Enfin, 9,9\% de l'échantillon ne s'identifie pas à une sous-culture ou s'identifie à une sous-culture autre que celles mentionnées ci-dessus. Étant donné que certaines de ces catégories regroupent un nombre trop petit d'individus pour effectuer une analyse statistique avec une puissance raisonnable, nous avons préféré regrouper les sous-cultures en deux catégories générales, c'est-à-dire les conventionnelles $(71,2 \%)$ et les non conventionnelles $(28,8 \%)$. Il ressort également que la participation au taxage varie de façon statistiquement significative selon ces deux sous-cultures d'identification $\left(X^{2}=9,66 ; p=0,002\right)$. Ainsi, parmi les adolescents conventionnels, $11 \%$ seulement disent avoir taxé, alors que $23 \%$ des 
adolescents non conventionnels sont dans la même situation. Par conséquent, l'hypothèse selon laquelle la participation au taxage serait le résultat direct de l'identification à un groupe déviant de la sous-culture adolescente ne peut pas, à elle seule, expliquer cette différence de façon satisfaisante. Même si les taxeurs sont deux fois plus nombreux parmi les adolescents déviants, il n'en demeure pas moins qu'une proportion importante des taxeurs se recrute parmi les adolescents conventionnels. Dans le premier cas, il s'agit vraisemblablement d'une conduite déviante qui fait partie d'un style de vie déviant, alors que, pour les autres, il s'agit probablement d'une expérimentation isolée.

\section{La méthode utilisée}

Qu'un adolescent soit impliqué dans un acte de taxage d'un objet ou d'un droit de passage, il semble pertinent de se demander quelles sont les méthodes utilisées pour extorquer un bien ou de l'argent. Un peu plus de la moitié des taxeurs, soit $52 \%$, rapportent avoir utilisé une seule méthode pour extorquer d'autres adolescents; pour $38,7 \%$, il s'agit de la menace et, pour $6,5 \%$, il s'agit soit de coups et blessures ou de l'utilisation d'une arme. Les autres taxeurs révèlent combiner ces méthodes; il s'agit le plus souvent de menaces et de coups et blessures accompagnées de l'utilisation d'une arme, soit 30,7\%; viennent ensuite, dans l'ordre, les menaces armées $(11,3 \%)$, les menaces et les coups $(4,8 \%)$, les coups et blessures à la suite de l'utilisation d'une arme (1,6\%). Ainsi, les menaces sont la méthode d'extorsion la plus populaire, suivie de près par les menaces et les coups et blessures combinées avec l'utilisation d'une arme. Les adolescents taxés, selon les données fournies par Deguire (2000), déclarent deux fois moins souvent avoir détecté la présence d'une arme $(24 \%)$ que ne le font les taxeurs (50\%). Cette différence s'explique-t-elle par l'énervement des premiers ou par la vantardise des seconds? Nous ne saurions le dire.

En résumé, les taxeurs et les taxés s'entendent sur le fait que ce sont les menaces qui constituent la méthode principale pour extorquer un autre adolescent. Cette caractéristique du taxage en fait un acte délinquant moins grave que si une arme était utilisée dans la grande majorité des cas. Voyons si la méthode utilisée lors du taxage varie selon la cible de ce geste. Malgré le fait que les nombres soient restreints dans certaines catégories, le tableau 2 permet de constater que la méthode utilisée change selon la nature de la cible. Ainsi, lorsque de l'argent est extorqué ou 
qu'un bien d'une valeur monétaire élevée est convoité, l'utilisation d'une arme est signalée par les taxeurs dans au moins les deux tiers des cas. Par contre, lorsqu'il s'agit d'un objet prestigieux ou non, la menace suffit pour extorquer ce bien dans les deux tiers des cas. Les taxés corroborent ces observations, selon les données fournies par Deguire (2000). De plus, son étude montre que les droits de passage exigés sont moindres si la menace est utilisée, et plus élevés lorsque le taxeur agit avec l'aide d'une arme.

En somme, la méthode utilisée lors d'un taxage varie selon la nature de ce qui est taxé. Plus le gain est élevé, plus la méthode utilisée est complexe et inquiétante, parce qu'elle implique l'utilisation de la menace, des coups et d'une arme quelconque. Il en résulte que le taxage se situe, en termes de gravité, entre le vol d'une personne et le vol grave, selon les données de Fréchette et Le Blanc (1987). Le premier impliquait l'utilisation d'une arme dans $38 \%$ des cas et le second, dans $81 \%$ des cas; pour sa part, le taxage est perpétré avec une arme dans $50 \%$ des cas.

\section{La complicité}

Il est bien connu en criminologie que le passage à l'acte délinquant des adolescents est avant tout une activité de groupe. Fréchette et Le Blanc (1987) rapportent que la complicité domine pour tous les types de délits, sauf les attaques contre les personnes; elle varie de $44 \%$ pour ces délits à $90 \%$ pour les vols avec effraction, et elle se situe à plus de $66 \%$ pour huit de douze types de délits analysés par ces chercheurs.

TABLEA U 2

La méthode utilisée selon la nature des objets taxés et la valeur des droits de passage

\begin{tabular}{|l|c|c|c|c|}
\hline \multirow{2}{*}{\multicolumn{1}{c|}{ Objet et \$ }} & \multicolumn{3}{|c|}{ Méthodes } & \multirow{2}{*}{ Coups } \\
\cline { 2 - 5 } & Menace & $\begin{array}{c}\text { Utilisation } \\
\text { et blessures } \\
\text { d'une arme }\end{array}$ & Total \\
\hline Prestigieux & $2(66,7 \%)$ & & $1(33,3 \%)$ & $3(4,6 \%)$ \\
\hline Non prestigieux & $8(72,7 \%)$ & & $3(27,3 \%)$ & $11(16,9 \%)$ \\
\hline Argent & $3(18,8 \%)$ & $2(12,5 \%)$ & $11(68,8 \%)$ & $16(24,6 \%)$ \\
\hline Valeur \$ significative & $2(14,3 \%)$ & $2(14,3 \%)$ & $10(71,4 \%)$ & $14(21,5 \%)$ \\
\hline \$ non précisé & $8(38,1 \%)$ & $4(19,0 \%)$ & $9(42,9 \%)$ & $21(32,3 \%)$ \\
\hline Total & $23(35,4 \%)$ & $8(12,3 \%)$ & $34(52,3 \%)$ & $65(100,0 \%)$ \\
\hline
\end{tabular}


Le taxage est également une activité de groupe, puisque 71,7\% des taxeurs qui ont extorqué un objet disent avoir agi en complicité (cette proportion est de 70,8\% lorsqu'il s'agit d'exiger un droit de passage). Par contre, le nombre de complices se répartit à peu près également entre un complice $(26,4 \%)$, deux complices $(18,9 \%)$ et trois complices ou plus $(26,4 \%)$, lorsqu'il s'agit d'extorquer un objet; ces proportions different peu lorsqu'il s'agit de percevoir un droit de passage, respectivement 33,3\%, $16,7 \%$ et $20,8 \%$ (les taxés confirment ces observations (Deguire, 2000)).

Le taxage est donc une activité délinquante qui ressemble aux autres par la fréquence de la complicité. De plus, Deguire (2000) montre que la complicité varie selon la méthode utilisée. Le nombre de complices augmente avec la gravité du geste. La menace est surtout utilisée lorsque le taxeur agit seul ou avec un seul complice, tandis qu'une arme est plus souvent présente lorsque le nombre de complices est de deux ou plus.

\section{Les lieux du taxage}

Le taxage est souvent présenté comme une activité principalement perpétrée en rapport avec la fréquentation scolaire. Nos données ne confirment pas cette caractéristique du taxage. En effet, 17,3\% seulement des taxeurs rapportent avoir extorqué un objet à l'école, contre $75 \%$ dans la rue ou un autre lieu public (ces proportions sont respectivement de $8 \%$ et $76 \%$ pour un droit de passage). Parmi ces dernières activités, 55,8\% ont eu lieu dans la rue; dans certains cas, mais pas toujours, en allant ou revenant de l'école (60\% pour un droit de passage). Les victimes corroborent cette description de la localisation du taxage rapportée par les auteurs (Deguire, 2000). Quant au rapport entre les lieux du taxage et la méthode utilisée, Deguire (2000) montre que la menace domine à l'école, alors que la présence d'une arme est beaucoup plus fréquente dans la rue, qu'il s'agisse d'extorquer un objet ou d'exiger un droit de passage.

\section{Conclusion}

La société, par l'entremise des médias, s'est montrée préoccupée par le phénomène du taxage. Elle le décrivait comme nouveau, généralisé, associé à la fréquentation scolaire, empreint de violence grave et comme étant le fait de catégories sociales spécifiques. Nos données ne confirment pas cette description du taxage, ni du point de vue des auteurs, ni du point de vue des victimes. 
En effet, le taxage n'est pas un phénomène délinquant nouveau, puisque plusieurs adultes se rappellent en avoir été victimes au cours de plusieurs décennies antérieures. De plus, c'est un phénomène comptabilisé en France depuis une vingtaine d'années, et, somme toute, il progresse peu (Debarbieux, 1996). Par ailleurs, le taxage, selon les données rapportées dans cet article, n'est pas généralisé, puisqu'on a calculé, à partir d'une enquête auprès d'un échantillon représentatif d'adolescents, que ce geste est effectué par $14 \%$ d'entre eux, mais seulement par environ $9 \%$ au cours des douze derniers mois. Ces proportions se comparent à celles obtenues dans les enquêtes américaines et européennes citées. À la question de savoir si le taxage récent variait selon certaines caractéristiques des adolescents, on a trouvé que le taxage était effectué majoritairement par des garçons de 15 à 16 ans. Ce résultat est celui que l'on retrouve en comparant les filles et les garçons pour toutes les formes de délinquance (Fréchette et Le Blanc, 1987; Lanctôt et Le Blanc, 2002).

Il serait trop facile de conclure que le taxage est une forme de délinquance qui se distingue peu des autres formes, compte tenu des données sur sa prévalence et sa distribution dans diverses catégories sociales. C'est pourquoi nous nous sommes intéressés aux modalités du passage à l'acte pour mieux apprécier si cette catégorie de gestes émane de la différenciation de la sous-culture adolescente en sous-groupes ou si elle constitue une manifestation analogue aux autres actes délinquants.

Le taxage n'apparaît pas uniquement comme une résultante de la différenciation de la sous-culture adolescente en catégories distinctes. En effet, très peu d'événements de taxage concernent des objets de prestige social pour l'une ou l'autre catégorie de cette sous-culture. Le taxage concerne davantage l'obtention d'argent et d'objets divers, sans oublier les droits de passage, beaucoup plus rares. Ainsi, bien que la moitié des taxeurs s'identifie à la culture dominante, où règne une forte publicité qui incite ces adolescents à consommer, il n'en reste pas moins que ceux-ci taxent principalement de l'argent sans que ce soit contre un droit de passage et il est plutôt rare que des objets prestigieux soient taxés. On pourrait envisager que les adolescents taxent de l'argent dans le dessein de se procurer des objets prestigieux, mais cela impliquerait un taxage répétitif qui ne se manifeste que rarement lorsque l'on considère la fréquence actuelle du taxage. Ainsi, il n'est pas justifié, à partir de nos résultats, de définir le taxage uniquement comme une forme particulière de vol provoqué par des pressions sociales provenant de la société de consommation. Le taxage semble se définir comme un vol avec violence, plutôt qu'une manifestation 
de délinquance issue des exigences de la société de consommation. Il semble donc faire partie du syndrome de la déviance qui représente un style de vie marginal. En effet, Deguire (2000) compare les taxeurs avec des délinquants non taxeurs : il ressort que les uns et les autres présentent la même variété de conduites délinquantes et déviantes.

Le taxage se manifeste clairement comme un acte délinquant ordinaire parce qu'il est accompli avec des complices et parce que son niveau de gravité est habituellement léger compte tenu de la méthode utilisée le plus fréquemment, à savoir la menace. Cette forme de taxage est celle que l'on retrouve essentiellement chez les adolescents conventionnels. Néanmoins, dans une faible proportion de cas et particulièrement chez les taxeurs qui appartiennent à une sous-culture déviante, il s'agit d'un acte complexe et violent. Il est complexe parce qu'il implique plusieurs méthodes, menaces, violence physique et présence d'une arme. Il est violent et dangereux pour la victime, par la présence de complices et cette accumulation de méthodes. Par ailleurs, le taxage n'apparaît pas comme un acte délinquant qu'il faut associer à la fréquentation scolaire car seule une faible proportion de ces actes a lieu à l'école. C'est une activité de rue qui prend place à différents moments de la journée. Les données obtenues remettent en question la stratégie de prévention qui est habituellement privilégiée, c'est-à-dire centrer les actions de prévention sur le phénomène du taxage. En effet, compte tenu de l'endroit où sont commis les actes et du caractère ludique et commun de beaucoup d'entre eux ou de leur intégration à un style de vie délinquant, il apparaît davantage pertinent de privilégier la prévention de la délinquance en général plutôt que la prévention d'une forme particulière de délinquance. La délinquance, dont le taxage, ne se manifeste pas, tout au moins chez les adolescents, comme une forme spécialisée de conduite déviante, mais comme un syndrome (Le Blanc et Bouthillier, 2002). Le taxeur, même occasionnel, est aussi occasionnellement un voleur à l'étalage ou un vandale.

La présente étude constitue un premier pas pour clarifier la conception d'un phénomène qui fait beaucoup parler, mais dont la nature du passage à l'acte n'avait pas été documentée de façon empirique. Ainsi, le taxage ne se présente pas comme une forme spécifique de conduite délinquante. Cette conduite s'apparente plutôt à du vol avec violence. Toutefois, certaines questions demeurent : les taxeurs se recrutent-ils parmi les récidivistes ou les petits délinquants? Présentent-ils des caractéristiques sociales et personnelles spécifiques? Comment les taxeurs se distinguent-ils des autres délinquants et des adolescents conventionnels? 


\section{Annexe : Questionnaire sur le taxage}

Pour chacun des comportements de taxage, les questions suivantes ont été posées aux auteurs et aux victimes d'un acte de taxage :

- L'as-tu déjà fait?

$$
\text { 1- oui 2- non }
$$

\section{SI OUI}

- à quel âge l'as-tu fait pour la première fois? ans

- Au cours des 12 derniers mois, as-tu ...?

1- jamais 2- une ou deux fois

3- plusieurs fois 4- très souvent

- Quelle chose as-tu demandée la dernière fois :

(Précise l'objet en question et sa marque de commerce. Par exemple : une tablette de chocolat de Cadbury).

- Avec qui l'as-tu fait la dernière fois?

1 - seul

2- avec une autre personne

3- avec deux autres personnes

4- avec plus de deux autres personnes

- Où l'as-tu fait la dernière fois?

1 - à l'école où tu suis tes cours

2- à une autre école que celle où tu suis tes cours

3- dans un lieu public (parc, arcade, ...)

4- dans la rue

5 autre : (Précisez)

Les comportements de taxage suivants ont été investigués :

1. Avoir menacé quelqu'un de le battre pour qu'il (elle) te donne quelque chose qui lui appartenait?

2. Avoir donné des coups de poing ou des coups de pied à quelqu'un pour qu'il (elle) te donne quelque chose qui lui appartenait? 
3. Avoir utilisé une arme (bâton, couteau, fusil, roches,...) pour obtenir quelque chose qui appartenait à quelqu'un?

4. Avoir menacé quelqu'un de le battre pour qu'il (elle) te donne de l'argent en échange d'un droit de passage?

5. Avoir donné des coups de poing ou des coups de pied à quelqu'un pour qu'il (elle) te donne de l'argent en échange d'un droit de passage?

6. Avoir utilisé une arme (bâton, couteau, fusil, roches,...) pour que quelqu'un te donne de l'argent en échange d'un droit de passage?

\section{Références}

Actualités Québec (1999).

Bianki, J., \& Lampron, N. (1998). Brisons le mur du silence; guide de prévention sur le taxage, la violence, l'agression sexuelle et les droits de la personne. Ottawa : Ministère du Solliciteur général de Canada.

Buidin, G., Petit, S., Philippot, P., \& Born, M. (2000). Violences à l'école : enquête de victimisation dans l'enseignement secondaire de la communauté française de Belgique. Louvain-la-Neuve : Université catholique de Louvain et Université de Liège.

Code criminel annoté et lois connexes (1991). Cowansville : Les éditions Yvon Blais.

Conseil scolaire de Montréal (1999). Déclaration de la clientèle. Montréal : Conseil scolaire de Montréal.

Debarbieux, E. (1996). La violence en milieu scolaire, état des lieux. Paris : ESF.

Debarbieux, E. (2001). La violence en milieu scolaire, dix approches en Europe. Paris : ESF.

Debarbieux, E., Dupuch, A., \& Montaya, Y. (1997). Pour en finir avec le handicap «socioviolent» : une approche comparative de la violence en milieu scolaire. In B. Charlot \& J-C. Émin (eds), Violences à l'école, état des savoirs. Paris : Armand colin.

Deguire, A.-E. (2000). Le taxage chez les adolescents montréalais : prévalence, passage à l'acte et caractéristiques des taxeurs. Montréal : Mémoire de maîtrise inédit, École de psychoéducation, Université de Montréal.

Fréchette, M., \& Le Blanc, M. (1987). Délinquance et délinquants. Québec : Morin.

Garofalo, J., Siegel, L., \& Laub, J. (1987). School-related victimizations among adolescents : An analysis of National Crime Survey (NCS) narratives. Journal of Quantitative Criminology, 3 (4), 321-338.

Kingery, P.M., Coggeshall, M.B., \& Alford, A.A. (1998). Violence at school : Recent evidence from four national surveys. Psychology in the Schools, 5 (3), 247-258.

Kuther, T.L., \& Fisher, C.B. (1998). Victimization by community violence in young adolescents from a suburban city. Journal of Early Adolescence, 18 (1), $53-76$. 
La Presse. Dossier présenté les 13-14-15 décembre 1998.

Lanctôt, N., \& Le Blanc, M. (2002). Explaining adolescent females' involvement in general deviance: Towards an integration of theoretical perspectives. Crime and justice, (à paraître).

Le Blanc, M. (1996). Mesures de l'adaptation sociale et personnelle pour les adolescents québécois. Montréal : École de psychoéducation, Groupe de recherche sur les adolescents en difficulté, Université de Montréal.

Le Blanc, M. (1999). Comportements et adolescents violents, un phénomène spécifique. In J. Proulx, M. Cusson \& M. Ouimet (eds), Les violences criminelles. Montréal : Presses de l'Université Laval.

Le Blanc, M., \& Bouthillier, C. (2002). A developmental test of the general deviance syndrome with adjudicated girls and boys using hierarchical confirmatory factor analysis. Criminal behavior and mental health, (à paraître).

Lejeune, R. (1977). The management of a mugging. Urban Life, 6 (2), 123-148.

Olweus, D. (1993). Bullying at school: What we know and what we can do. Oxford : Cambridge.

Poupart, P., \& Duval, A. (1996). Opération «taxage». Montréal : Les Centres jeunesse de Montréal.

Service de Police de la Communauté Urbaine de Montréal. (1995). Bilan annuel 1995.

Statistique Canada (1993-1994). Statistiques sur les tribunaux de la jeunesse. Centre Canadien de la statistique juridique.

Statistique Canada (1994-1995). Statistiques sur les tribunaux de la jeunesse. Centre Canadien de la statistique juridique. 